\title{
Alterations in spatial memory and anxiety in the MAM E17 rat model of hippocampal pathology in schizophrenia
}

\author{
Francois Gastambide • Amy M. Taylor • Clare Palmer • \\ Heta Svard • Maija Karjalainen • Sanna K. Janhunen • \\ Mark Tricklebank • David M. Bannerman
}

Received: 14 October 2014 / Accepted: 29 December 2014 / Published online: 31 January 2015

(C) Springer-Verlag Berlin Heidelberg 2015

\begin{abstract}
Adult rats exposed to methylazoxymethanol acetate (MAM) at embryonic day 17 (E17) display robust pathological alterations in the hippocampus. However, discrepancies exist in the literature regarding the behavioural effects of this pre-natal manipulation. Therefore, a systematic assessment of MAM E17-induced behavioural alterations was conducted using a battery of dorsal and ventral hippocampusdependent tests. Compared to saline controls, MAM E17treated rats displayed deficits in spatial reference memory in both the aversive hidden platform watermaze task and an appetitive Y-maze task. Deficits in the spatial reference memory watermaze task were replicated across three different cohorts and two laboratories. In contrast, there was little, or no, effect on the non-spatial, visible platform watermaze task or an appetitive, non-spatial, visual discrimination task, respectively. MAM rats were also impaired in the spatial novelty preference task which assesses short-term memory, and displayed reduced anxiety levels in the elevated plus maze task. Thus, MAM E17 administration resulted in abnormal spatial information processing and reduced anxiety in a number of hippocampus-dependent behavioural tests, paralleling the effects of dorsal and ventral hippocampal lesions, respectively. These findings corroborate recent pathological and physiological studies, further highlighting the usefulness of MAM E17
\end{abstract}

F. Gastambide $(\bowtie) \cdot$ M. Tricklebank

Lilly Centre for Cognitive Neuroscience, Eli Lilly \& Co. Ltd, Erl

Wood Manor, Windlesham, UK

e-mail: gastambidefr@lilly.com

A. M. Taylor $\cdot$ C. Palmer $\cdot$ D. M. Bannerman

Department of Experimental Psychology, University of Oxford, Oxford, UK

H. Svard · M. Karjalainen · S. K. Janhunen

Orion Corporation, Orion Pharma, R\&D, CNS Research,

Turku, Finland as a model of hippocampal dysfunction in at least some aspects of schizophrenia.

Keywords Methylazoxymethanol acetate $\cdot$ Hippocampus · Spatial learning $\cdot$ Anxiety $\cdot$ Emotionality $\cdot$ Rats

\section{Introduction}

Schizophrenia is a complex and heterogeneous disorder, associated with profound deficits in a wide variety of cognitive domains, including attention, memory and executive function (Heinrichs and Zakzanis. 1998; Nuechterlein et al. 2004). Structural and functional alterations in cortical and subcortical areas are thought to mediate these deficits (Antonova et al. 2004; Reichenberg and Harvey. 2007). Evidence for the importance of the hippocampus in the pathology of schizophrenia is compelling (for review, see Harrison. 2004; Tamminga et al. 2010). Most of these findings derive from post-mortem, brain imaging and neuropsychological studies, and range from reduced hippocampal volume (Lawrie and Abukmeil. 1998; Nelson et al. 1998; Wright et al. 2000; Honea et al. 2005), abnormal neuronal organisation (Kovelman and Scheibel. 1984; Heckers and Konradi. 2002; Zhang and Reynolds. 2002) and altered synaptic transmission (Tsai et al. 1995; Gao et al. 2000; Medoff et al. 2001; Harrison et al. 2003; Schobel et al. 2009) to deficits in hippocampal-dependent memory tasks and changes in task-related functional activation of the hippocampus (Heckers et al. 1998; Aleman et al. 1999; Weiss and Heckers. 2001; Cirillo and Seidman. 2003; Jessen et al. 2003; Leube et al. 2003; Hanlon et al. 2006; Ongur et al. 2006; Achim et al. 2007; Boyer et al. 2007; Folley et al. 2010; Stone and Hsi. 2011). 
Evidence consistent with hippocampal alterations in schizophrenia also comes from animal models, including not only neonatal ventral hippocampal damage but also the embryonic day 17 methylazoxymethanol acetate (MAM E17) rodent model which exhibits robust hippocampal pathology. Through selective disturbance of proliferation and migration of neuronal precursor cells, MAM E17 treatment interferes with the development of cortical and subcortical brain regions, and results in a series of structural and functional alterations that are consistent with those observed in schizophrenia (Moore et al. 2006; Lodge and Grace. 2009). Changes in the hippocampus include volume reduction, aberrant neuronal organisation and excitability, deficits in synaptic transmission and synaptic plasticity, and abnormal neuronal oscillatory activity (Gourevitch et al. 2004; Moore et al. 2006; Penschuck et al. 2006; Lodge and Grace. 2007; Matricon et al. 2010; Chin et al. 2011; Hradetzky et al. 2012; Phillips et al. 2012a, b; Sanderson et al. 2012; Snyder et al. 2013). Alongside these robust anatomical and physiological alterations, a number of studies have looked at the effects of MAM E17 treatment in behavioural paradigms assessing hippocampus-dependent spatial memory (Gourevitch et al. 2004; Flagstad et al. 2005; Le Pen et al. 2006; Featherstone et al. 2009; Hazane et al. 2009; Snyder et al. 2013). While there appear to be consistent, robust and reliable spatial working/short-term memory deficits in MAM E17 rats (Gourevitch et al. 2004; Featherstone et al. 2009; Hazane et al. 2009), the picture for associative, long-term spatial reference memory tasks is less clear cut.

Spatial reference memory has been assessed primarily using the fixed location, hidden escape platform version of the open-field Morris watermaze task but with mixed results. For example, whereas one study demonstrated a clear impairment in MAM E17 rats during watermaze acquisition (Hazane et al. 2009), another study found no impairment whatsoever (Flagstad et al. 2005). In both studies, latencies and path lengths were reported during acquisition, but there was no explicit test of spatial memory abilities such as a probe test in which the platform is removed from the pool and the rats allowed to swim freely for a short-period of time to see where they spend their time searching. It is important to point out that animals can show sizeable improvements in performance during watermaze training (i.e. reductions in latencies and/or path lengths) without necessarily learning anything about the actual allocentric spatial location of the platform (e.g. they can simply learn to swim away from the side walls). Thus, an explicit test of spatial memory is desirable. In a more recent study, Snyder and colleagues (2013) did perform probe tests at the end of watermaze training and found that although MAM E17 animals were impaired during acquisition (in terms of escape latencies) when tested at either juvenile (P12-P21) or adolescent ages (P28-P45), a different picture emerged from the probe tests. Although juvenile MAM E17 rats were impaired, MAM E17 rats were not impaired if tested as adolescents. In addition, none of these aforementioned studies have included a non-spatial, control task such as a visible platform task to exclude the possibility that any watermaze deficit could be due to disruption of sensorimotor or motivational processes rather than a deficit in spatial memory per se. Furthermore, all of these studies have used the aversive watermaze task and the effects of MAM E17 on a non-aversive, associative spatial reference memory task have not been examined. Thus, the specificity and generality of any spatial reference memory deficit have not been assessed.

The rationale for the present study therefore was to reinvestigate MAM E17-induced behavioural alterations, using a battery of hippocampus-dependent spatial memory tests, assessing both short-term and long-term spatial memory, and using both aversive and non-aversive testing paradigms. Specifically, spatial memory performance was assessed using the aversive open-field Morris watermaze spatial reference memory task, the appetitive Y-maze spatial reference memory test and the spontaneous Y-maze spatial novelty preference test which assesses short-term memory. Appropriate hippocampus-independent, non-spatial control tests were also included. While lesion studies have demonstrated an important role for the dorsal hippocampus in spatial memory, the ventral hippocampus has been more associated with anxiety (Bannerman et al. 2002, 2003, 2004, 2014; Kjelstrup et al. 2002; McHugh et al. 2004; Fanselow and Dong. 2010). Therefore, ventral hippocampal dysfunction was also assessed using the elevated plus maze test of anxiety.

\section{Materials and methods}

\section{Subjects}

Time-mated Sprague-Dawley dams (Charles River, UK) were dosed intraperitoneally at embryonic day 17 with $22 \mathrm{mg} / \mathrm{kg}$ of methylazoxymethanol acetate (MAM) or $0.9 \%$ saline $(1 \mathrm{ml} /$ $\mathrm{kg}$ ). At postnatal day 28 , litters were weaned. Male offspring were kept and housed with non-littermates from the same treatment group, in standard housing conditions (two to four rats per cage, light phase $0700-1900$, controlled temperature and humidity). Rats were 4-8 months old at the time of behavioural testing and had ad libitum access to food and water, except prior to the appetitive Y-maze spatial reference memory and T-maze visual discrimination tasks, during which they were maintained at $85 \%$ of their free-feeding weight. All experiments were conducted during the light phase, by an experimenter unaware of the rats' group assignments and in accordance with the European Communities Council Directive 86/609/EEC regarding the care and use of animals for experimentation. 


\section{Order of testing}

A total of 57 saline and 63 MAM rats were used in the present study. Experiments were performed across two separate laboratories (University of Oxford, UK in collaboration with Eli Lilly, and Orion Pharma, Finland). Two cohorts of animals were used in each laboratory. Two cohorts of 24 and 8 animals per group, respectively, were used by Orion Pharma in Finland to assess MAM E17 effects in the Morris watermaze hidden platform spatial reference memory test and their within-lab reproducibility.

In addition, behavioural performance on this task and other tests of spatial memory, non-spatial memory control tasks and a test of anxiety was also assessed in a different laboratory (Oxford, UK). The first cohort of 12 saline and 15 MAM rats used by Eli Lilly/Oxford, UK was tested in five different behavioural paradigms: the fixed location, hidden platform, spatial reference memory Morris watermaze task, the elevated plus maze test of anxiety, spontaneous spatial novelty preference in an enclosed Perspex Y-maze (a test of short-term spatial memory), appetitively motivated spatial reference memory in the elevated Y-maze, and non-spatial, visual discrimination learning in an enclosed T-maze. The second cohort of 13 saline and 16 MAM rats was used for the assessment of anxiety levels in experimentally naïve animals using the elevated plus maze, and was then tested in the non-spatial visible platform Morris watermaze task (see Table 1 for summary of behavioural experiments performed by all the four separate cohorts of MAM E17 rats).

Table 1 Summary of behavioural experiments for all four cohorts of MAM 17 rats

Orion cohort $1(n=24)$
1. Spatial reference memory in the Morris watermaze
Orion cohort $2(n=8)$
1. Spatial reference memory in the Morris watermaze
Oxford cohort $1(n=12-15)$ :
1. Spatial reference memory in the Morris watermaze
2. Elevated plus maze
3. Spontaneous spatial novelty preference test in an enclosed Y-maze
4. Appetitive, spatial reference memory Y-maze task
5. Appetitive, non-spatial (visual discrimination) T-maze task
Oxford cohort 2 ( $n=13-16)$
1. Elevated plus maze
2. Non-spatial (visible platform) version of the watermaze task

The table includes the total number of rats used per group per cohort as well as the temporal sequence in which the different behavioural experiments were performed
Hidden platform spatial reference memory in the Morris watermaze

\section{Orion pharma protocol}

Testing was conducted in a black circular tank of $1.5 \mathrm{~m}$ diameter and containing clear water at a temperature of $21 \pm 1{ }^{\circ} \mathrm{C}$. Rats were trained for 5 days (three trials per day). The platform was located at the centre of the NE quadrant of the pool. During each trial, rats were placed into the pool facing the side wall at one of three start locations (NW, SE and SW; chosen randomly across trials) and allowed to swim until they found the platform or for a maximum of $60 \mathrm{~s}$. Any rat that failed to find the platform within $60 \mathrm{~s}$ was guided to its location by the experimenter and allowed to remain on the platform for $30 \mathrm{~s}$. The rat was left for another $30 \mathrm{~s}$ outside the pool in a cage before commencing the next trial.

\section{Eli Lilly/Oxford protocol}

The protocol used at Oxford University was similar in many respects to the one described above. However, there were some important differences. Most notably, we included (i) a probe (transfer) test to assess spatial memory performance $24 \mathrm{~h}$ after the last spatial training trial, and (ii) a non-spatial, visible platform control task which was run in separate groups of rats.

Animals had no swim pre-training before testing. Testing was conducted in a large circular tank (diameter $2.0 \mathrm{~m}$, depth $0.6 \mathrm{~m}$ ) containing water at a temperature of $25 \pm 1^{\circ} \mathrm{C}$, and to a depth of $0.3 \mathrm{~m}$. To escape from the water, rats had to find a hidden escape platform (diameter $10 \mathrm{~cm}, 1 \mathrm{~cm}$ below the water surface) situated in a fixed spatial location. The water was made opaque by the addition of 21 of semi-skimmed milk, which not only prevented the subjects from seeing the platform but also allowed efficient tracking of swim paths. The pool was surrounded by prominent distal extra-maze cues that could be used as landmarks (shelves, racks of equipment, posters on walls, etc.). Swim paths were tracked by a video camera mounted in the ceiling, and relayed to a computer for image analysis using specialised software (HVS Image Analyse, Hampton, UK). The $x$ and $y$ coordinates of each rat's position were sampled in real time at $10 \mathrm{~Hz}$ by an Acorn computer, using a software that provided measures such as latency, path length and swim speed during acquisition, and the time spent in each quadrant of the pool during the probe/ transfer tests. Rats were trained for 8 days (four trials per day). The platform was located at the centre of either the NW or SE quadrant of the pool. The number of rats trained to each platform location was counterbalanced with respect to treatment group. During each trial, rats were placed into the pool facing the side wall at one of eight start locations (arbitrarily designated N, S, E, W, NE, NW, SE and SW; chosen randomly 
across trials) and allowed to swim until they found the platform or for a maximum of $90 \mathrm{~s}$. Any rat that failed to find the platform within $90 \mathrm{~s}$ was guided to its location by the experimenter and allowed to remain on the platform for $30 \mathrm{~s}$ before commencing the next trial. On completion of four daily trials, rats were dried and then returned to their home cages.

Twenty-four hours after the fourth and eighth days of testing, (i.e. after trials 16 and 32, respectively), probe/transfer tests were conducted to determine the extent to which the rats had learned about the location of the platform. The platform was removed from the pool, and each rat was allowed to swim freely for $60 \mathrm{~s}$. The percentage of time spent, and the number of (would-be) platform crossings, in each quadrant were recorded for each animal. In the analysis of the distribution of time spent searching the four quadrants, the numerator term in the degrees of freedom was reduced by one to control for the fact that the four quadrant dwell times were not independent of each other. In the analysis of the number of annulus (platform) crossings in each quadrant, an accuracy score was calculated as follows: number of crossings in the goal platform annulus-(number of crossings in all 3 other would-be platform annuli/3).

\section{Visible platform learning in the Morris watermaze}

The visible platform task was performed at Oxford University on a separate group of rats and used as a control for the hidden platform task. This variant of the task involves similar sensorimotor and motivational demands but without the need for spatial information processing. Rats were trained for 3 days (four trials per day) to find a visible escape platform (diameter $10 \mathrm{~cm}, 1 \mathrm{~cm}$ above the water surface), which had a variable spatial location. The escape location was indicated by placement of a visible, black-and-white-striped cylinder directly above the platform. The extra-maze spatial cues were not occluded during visible platform training. To ensure that there was no spatial solution to the task, both the platform position and the start location were varied randomly from trial to trial.

\section{Spatial reference memory in the Y-maze}

Appetitively motivated spatial reference memory was examined using a Y-maze made of black painted wood. It had a central polygonal area $9 \mathrm{~cm}$ in diameter, to which three identical arms were attached (each $70 \times 9 \times 5 \mathrm{~cm}$ ). A small metal food well was glued to each arm $5 \mathrm{~cm}$ from the distal end. The maze was elevated $70 \mathrm{~cm}$ above the floor on a central stand, on which the entire maze could be rotated. The room contained a variety of prominent features, which served as distal spatial cues. Rats were familiarized to the maze in their colony holding room (i.e. not the testing room), until they were running freely on the maze and readily consuming pellet rewards from the food wells.
For spatial memory testing, a goal arm (defined according to its allocentric position relative to the room cues) was designated for each rat. Goal arms were counterbalanced with respect to treatment group such that approximately equal numbers of saline and MAM animals were trained to each of the three arms. The start arm for each trial was determined by pseudorandom sequence (with equal numbers of starts from each of the other two arms in any one session, and no more than three consecutive starts from the same arm). The maze was rotated $120^{\circ}$ in a clockwise or anticlockwise direction at random intervals during testing, to prevent the rat identifying the rewarded arm by any olfactory, visual or tactile cues unique to a particular arm. For each trial, the food well on the goal arm was filled with a $45 \mathrm{mg}$ sucrose pellet reward, and the rat was placed at the distal end of a start arm, facing outwards. A correct choice was scored when the rat entered the goal arm directly. If the rat chose incorrectly, it was immediately removed from the maze and then returned to its home cage. Rats were trained for 6 days (10 trials per day) with an inter-trial interval (ITI) of approximately $10 \mathrm{~min}$. During the final 10 trials, the pellet reward was delivered into the food well "only" after rats had made a choice (post-choice baiting). This was to ensure that rats were unable to solve the task by smelling the food reward.

\section{Visual discrimination learning in the T-maze}

The T-maze visual discrimination task was used as a control for the appetitive Y-maze spatial reference memory task, involving similar sensorimotor and motivational demands but without the need for spatial information processing. The enclosed, dark grey, wooden T-maze had a start arm and two interchangeable goal arms; one painted grey arm and one black and white striped arm (each $60 \times 10 \times 30 \mathrm{~cm}$ ). An aluminium food well was attached $3 \mathrm{~cm}$ from the distal end of each of these two arms. Rats were familiarized to the maze with neutral/dark grey arms and then assigned to either one of two goal arms (i.e. the arm which will be rewarded during the task; grey vs. black/white stripes). Assignment to goal arms was counterbalanced across treatment groups. The right/left location of the rewarded goal arm varied from trial to trial (i.e. there was no spatial solution), and was determined according to a pseudorandom sequence (with equal numbers of right/left presentation and no more than three consecutive trials with the same correct location). For each trial, the food well in the goal arm was filled with a $45 \mathrm{mg}$ sucrose pellet reward, and the rat was placed at the distal end of the start arm, facing outwards. A correct choice was scored when the rat entered the assigned/correct goal arm directly. If the rat chose incorrectly, it was immediately removed from the maze and then returned to its home cage. Rats were trained for 8 days (10 trials per day) with an ITI of approximately $10 \mathrm{~min}$. During the final block of 10 trials, the pellet reward was 
delivered into the food well "only" after rats had made a choice (post-choice baiting). This was to ensure that rats were unable to solve the task by smelling the food reward.

Spatial novelty preference in the Y-maze

MAM and saline rats were also tested on a spontaneous, spatial novelty preference task to assess spatial short-term memory. This task provides a non-aversive, non-appetitive experimental context in which behaviour is driven by animals' natural exploratory drive. The Y-maze used was made of clear plastic and had three identical arms (each $49 \times 17 \times 27 \mathrm{~cm}$ ). Rats were pseudo-randomly assigned two arms (the 'start arm' and the 'familiar arm') to which they were exposed during the first 5-min phase of the task (the 'exposure phase'). The entrance to the third 'novel' arm was closed off by the presence of a large opaque Perspex block. At the end of the 5min period, the rat was removed from the maze, placed back in its home cage for $1 \mathrm{~min}$, following which the 'test' phase started. The Perspex block was removed. Rats were placed back into the start arm and allowed to explore the entire maze (i.e. all 3 arms) for $2 \mathrm{~min}$. Spatial novelty preference was assessed twice, in different rooms and with different arm assignments each time. For each test, the amount of time spent in each arm and the number of entries into each arm were recorded, during both the exposure and test phases. For the test phase, both a difference score (time in novel arm-time in familiar arm) and a discrimination ratio (time in novel arm/ (time in novel+familiar arms)) were calculated. Animals commonly display a marked preference for the novel arm during the test phase.

Anxiety in the elevated plus maze

Anxiety levels of MAM and saline rats were assessed using the elevated plus maze task. The maze consisted of two open grey arms (each $61 \times 10 \times 3 \mathrm{~cm}$ ) and two closed grey arms (each $61 \times 10 \times 30 \mathrm{~cm}$ ), and was arranged so that the two open arms were opposite each other. The maze had a $10 \times 10-\mathrm{cm}$ central square and was on a stand $39 \mathrm{~cm}$ above the ground.

Rats were placed individually into the centre of the maze, facing into one of the open arms and were allowed to explore the maze freely. Each testing session lasted for $5 \mathrm{~min}$, during which the number of entries and time spent in each open or closed arm were measured. Rats were then removed and placed back into their home cages. Testing was performed in the two separate cohorts of rats. The first cohort (i.e. trained cohort) previously underwent watermaze testing whereas the second cohort did not undergo any behavioural testing before elevated plus maze testing (i.e. experimentally naïve cohort). The discrimination ratios of either time or entries into open vs. closed arms were calculated for each group as follows: time or entries into open arms/(time or entries into open+closed arms).
Statistical analyses

Behavioural data were analysed using repeated measures analysis of variance (RM-ANOVA) followed by post hoc comparisons (i.e. Tukey's test), where appropriate. The assumptions required for parametric ANOVA were checked (normality, equal variance) and data transformed where needed. In addition, one sample $t$ tests were also used to determine whether mean responses were significantly different from chance.

\section{Results}

Impaired aversive spatial reference memory in the watermaze

\section{Orion pharma}

MAM rats showed a profound and enduring impairment at acquiring the hidden platform watermaze task, in both cohorts of animals tested (Fig. 1a: cohort 1; Fig. 1b: cohort 2). Path lengths were analysed using a two-way RM-ANOVA, with treatment group as between-subject factor and trial block as a within-subject factor. These analyses revealed a significant effect of treatment group [cohort 1: $F(1,46)=72.47 ; p<0.001$; cohort $2: F(1,14)=65.57 ; p<0.001]$, a main effect of trial block [cohort 1: $F(4,184)=52.43 ; p<0.001$; cohort $2: F(4,56)=$ $34.38 ; p<0.001]$, and a significant group $\times$ block interaction [cohort 1: $F(4,184)=3.76 ; p<0.01$; cohort $2: F(4,56)=5.82$; $p<0.001]$. Importantly, performance on trial one of block one of training was the same for both groups [path length $(m)$; cohort 1: saline: $11.23 \pm 1.1$; MAM: $12.83 \pm 1.0 ; p>0.1$; cohort 2, saline: $12.83 \pm 0.9$; MAM: $12.68 \pm 2.1 ; p>0.1]$.

\section{Eli Lilly/Oxford}

The MAM E17 rats tested in Oxford also showed a profound and enduring impairment at acquiring the hidden platform watermaze task (Fig. 2a). Path lengths were analysed using a two-way RM-ANOVA, with treatment group as a betweensubjects factor, and trial block as a within-subjects factor. This analysis revealed a significant main effect of treatment group $[F(1,25)=41.25 ; p<0.001]$, a significant effect of block $[F(7,175)=30.58 ; p<0.001]$, but no group by block interaction $[p>0.1]$. Inspection of Fig. 2a shows that the MAM-treated rats were performing worse than the saline controls from the very first block of testing. Importantly, however, a separate analysis demonstrated that performance on trial one of block one of training was the same for both groups [path length $(m)$, saline: 25.7 \pm 2.2 ; MAM: $22.6 \pm 2.8 ; p>0.1]$. Further analyses including trial within block as a further within-subject factor revealed a main effect of trial $[F(3,600)=11.87 ; p<0.001]$, but no trial by group or trial by group by block interaction [both $p>0.1$. 

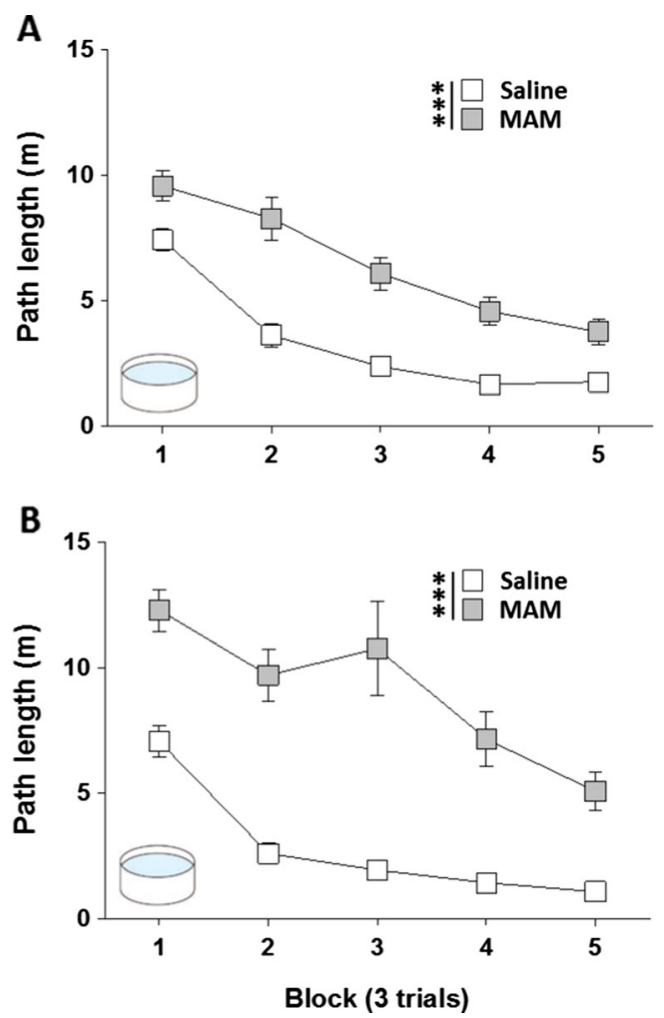

Fig. 1 MAM E17 exposure impaired hidden platform spatial reference memory in the Morris watermaze. Hidden platform Morris watermaze spatial reference memory was assessed by Orion Pharma (Finland) in two separate cohorts of rats: (a) cohort 1, $n=24$ per group; (b) cohort 2, $n=8$ per group. Results are presented as the mean \pm SEM of the path lengths travelled to reach the platform across each of the 3-trial training blocks for both MAM E17 rats (grey) and saline controls (white). *** $P<0.001$ vs. the saline-treated group

Spatial memory for the platform location was also assessed during two probe/transfer tests (conducted $24 \mathrm{~h}$ after trials 16 and 32), in which the escape platform was removed and the rats swam freely for $60 \mathrm{~s}$ (Fig. 2b). Analyses comparing the percentage of time spent in each quadrant by the two groups during both transfer tests revealed a main effect of quadrant in both probe tests $[F(2,75)=15.03 ; p<0.001$ in probe test 1 ; $F(2,69)=43.01 ; p<0.001$ in probe test 2], a trend towards a group by quadrant interaction in probe test $1[F(2,75)=2 ; p=$ $0.07]$, and a significant group by quadrant interaction in probe test $2[F(2,75)=4 ; p<0.05$ in test 2$]$. A separate analysis comparing the time spent in the training (goal) quadrant only revealed that each group showed a consistent spatial preference for the training quadrant when compared to chance performance [saline vs. $25 \%$ chance: $p<0.001$ in both tests; MAM vs. $25 \%$ chance: $p<0.01$ in test 1 and $p<0.001$ in test 2]. However, MAM-treated rats were significantly impaired compared to saline controls in terms of time spent in the training quadrant [MAM vs. saline: $p<0.01$ in test 1 and $p<0.05$ in test 2]. This deficit in spatial memory was confirmed by comparison of the accuracy score based on annulus crossings in each of the probe/transfer tests [MAM vs. saline: $p<0.05$ in test 1 and $p<0.001$ in test 2; saline vs. chance: $p<0.05$ in test 1 and $p<0.001$ in test 2; MAM vs. chance: $p>0.05$ in both tests].

Impaired aversive visible platform learning in the watermaze

A separate cohort of watermaze naïve MAM and saline rats was tested on the non-spatial visible platform version of the Morris watermaze task (Fig. 2c). Interestingly, MAM rats showed a small but significant impairment at acquiring this task. A two-way RM-ANOVA conducted on path lengths revealed a significant effect of block $[F(2,54)=133.13$; $p<0.001]$, a main effect of treatment group $[F(1,27)=9.02$; $p<0.01]$, and a trend towards group by block interaction $[F(2,54)=2.85 ; p=0.06]$. Again, visual inspection of Fig. $2 \mathrm{c}$ suggested that MAM rats were impaired during the first block of watermaze testing, although important analysis of trial one of block one of training revealed that performance was the same for both groups [path length $(m)$, saline: $19.2 \pm 1.5$; MAM: $23.2 \pm 1.5 ; p>0.05]$.

To determine whether the MAM rats were disproportionately impaired on the spatial version of the task, a further statistical analysis was conducted which compared path lengths to the platform for the first three training blocks on the respective tasks (spatial versus non-spatial, Oxford studies). Analysis of the two aversively motivated watermaze studies together suggested that MAM rats were disproportionately impaired in spatial learning compared to the visible platform task. Indeed, there was a significant group by task interaction $[F(1,52)=15.66 ; p<0.001]$. However, it is important to point out that the spatial and non-spatial (visible platform) watermaze tasks were conducted with different cohorts of rats, and were run as separate experiments, and so it is not strictly appropriate to combine these datasets in a single ANOVA. Therefore, caution is necessary when interpreting the results of such a combined analysis.

Impaired appetitive spatial reference memory in the Y-maze

To examine whether the MAM deficit extended to a nonaversively motivated, associative spatial memory task, MAM and saline rats were tested in the appetitive Y-maze spatial reference memory task (Fig. 3a). Consistent with the watermaze results, appetitively motivated spatial memory was also impaired in MAM rats. Percent correct choices were analysed using a two-way RM-ANOVA, with treatment group as a between-subject factor and trial block as a within-subject factor. These analyses revealed a main effect of treatment group $[F(1,24)=5.14 ; p<0.05]$, a significant effect of trial block $[F(5,120)=44.29 ; p<0.001]$, but no group by block interaction $[p>0.1]$. Post-choice reinforcement in block 6 confirmed that rats were not locating the food reward by virtue of its odour. 
A

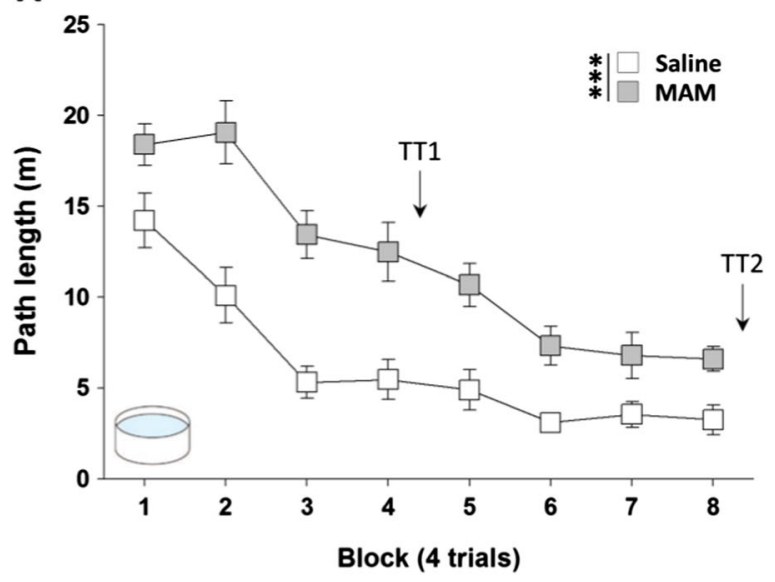

C

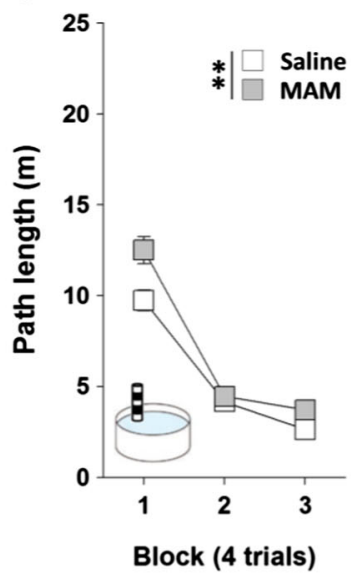

B Transfer test 1 (TT1), after 16 trials

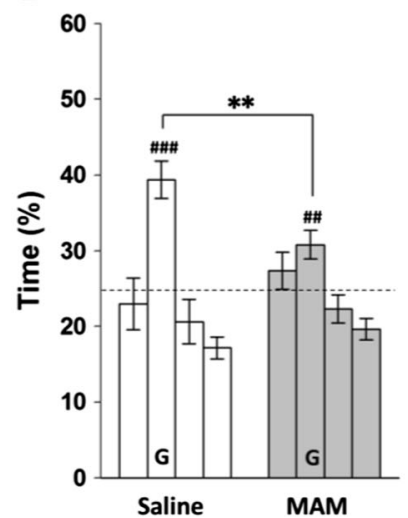

Fig. 2 MAM E17 exposure impaired both hidden (a-b) and visible platform learning (c) in the aversively motivated Morris watermaze. Hidden and visible platform Morris watermaze learning tasks were assessed in a separate laboratory (Oxford University in collaboration with Eli Lilly, UK) and in two separate cohorts of rats. a Results from the hidden platform learning task are presented as the mean \pm SEM of the path lengths travelled to reach the platform across each of the four-trial training blocks for both MAM E17 rats $($ grey, $n=15)$ and saline controls (white, $n=12$ ). b Transfer tests were conducted $24 \mathrm{~h}$ after the fourth (TT1)

Normal appetitive visual discrimination learning in the T-maze

MAM rats were not impaired in the acquisition of an appetitively motivated, non-spatial, visual discrimination learning T-maze task (Fig. 3b). A two-way RM-ANOVA with treatment group as a between-subject factor and trial block as a within-subject factor, revealed a significant effect of trial block $[F(7,168)=13.87 ; p<0.001]$ but no main effect of treatment group or group by block interaction [both $p>0.1]$. Again, post-choice reinforcement in block 8 confirmed that rats were not locating the food reward by virtue of its odour.

To further investigate the specificity of MAM-induced spatial deficits, another statistical analysis was conducted which compared percent correct choices for the first 6 training blocks

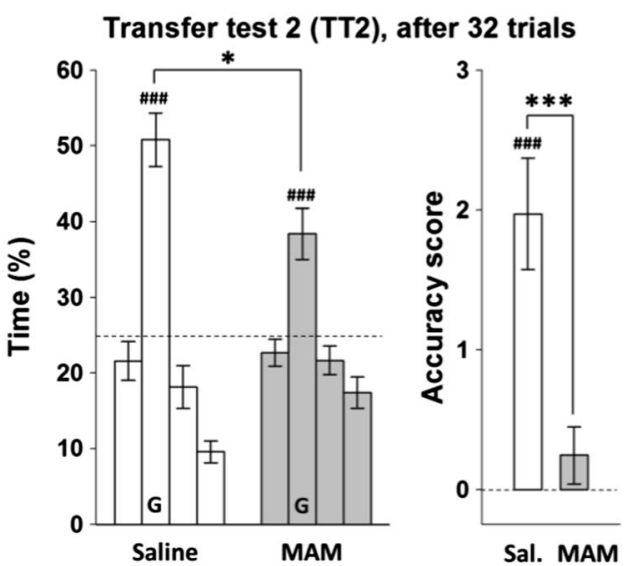

and eighth (TT2) block of training. Results are presented as the mean \pm SEM of the percentage of time spent in the goal quadrant $(G)$ and other three quadrants, as well as the platform crossing accuracy score for each transfer test. Dashed lines represent $25 \%$ chance level. c Results from the visible platform learning task are presented as the mean \pm SEM of the path lengths travelled to reach the platform across each of the four-trial training blocks for MAM E17 rats (grey, $n=16$ ) and saline controls (white, $n=13$ ). ${ }^{*} P<0.05 ;{ }^{*} P<0.01 ; * * * P<0.001$ vs. the saline-treated group. ${ }^{\#} P<0.05$;

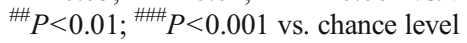

on the respective spatial and non-spatial (Y- and T-maze) tasks. Analysis of the two appetitively motivated maze studies together revealed a significant group by task interaction $[F(1$, $48)=4.5 ; p<0.05]$. Again, it is important to point out that the spatial and non-spatial (visible platform) appetitive maze tasks were conducted with different cohorts of rats, and were run as separate experiments, and so it is not strictly appropriate to combine these datasets in a single ANOVA. Again, we should be cautious when interpreting the results of this combined analysis.

Impaired spontaneous spatial novelty preference in the Y-maze

MAM rats were impaired in spatial novelty preference compared to saline-treated controls. The two tests were analysed 

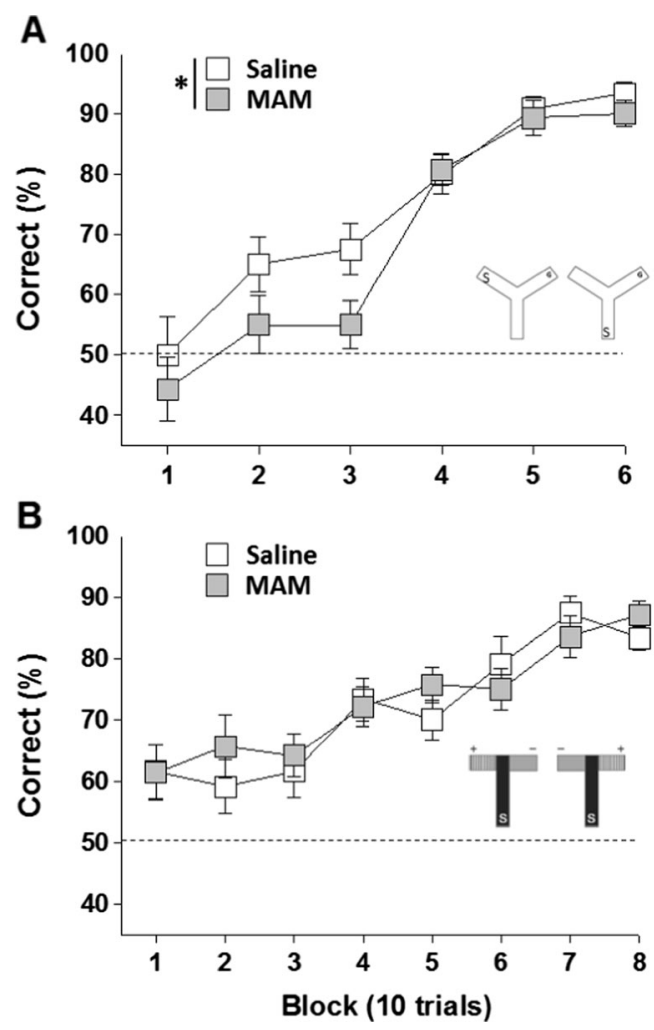

Fig. 3 MAM E17 exposure impaired spatial (a) but not visual discrimination learning (b) in the appetitively motivated Y-maze and Tmaze, respectively. Results are presented as the mean \pm SEM of the percentage of correct choices made by MAM E17 (grey, $n=14)$ and saline controls (white, $n=12$ ), across each of the 10-trial training blocks during either the Y-maze spatial reference learning task (a) or the T-maze visual discrimination learning task (b). Dashed lines represent $50 \%$ chance levels. ${ }^{*} P<0.05$ vs. the saline-treated group

together using three-way ANOVAs with test/room as a withinsubject factor, however, there was no main effect of test or group by test interaction [both $p>0.1$ ]. Although both treatment groups made a greater number of entries and spent more time in the novel arm vs. the familiar arm during the test phase (Fig. 4a-b), the mean time spent in the novel arm was significantly greater for saline controls [saline vs. MAM: $p<0.05$ ]. This was confirmed when comparing the difference scores (but not the discrimination ratios) of the time spent in the novel vs. familiar arm, respectively, against chance performance and between treatment groups (Fig. 4c-d). Both MAM and saline controls performed significantly above chance [difference score: saline $p<0.001$ and MAM $p<0.001$ vs. $0 \mathrm{~s}$ value; discrimination ratio: saline $p<0.001$ and MAM $p<0.001$ vs. 0.5 value], therefore suggesting robust novelty preference in both treatment groups. However, MAM rats spent a significantly shorter period of time exploring the novel arm compared to saline controls, as illustrated by the difference score [saline vs. MAM: $p<0.05$ ] but not the discrimination ratio which did not quite reach statistical significance [saline vs. MAM: $p>0.1$ ].
To investigate whether the difference in novelty preference between groups was due to MAM animals spending less time in the "to-be-familiar arm" during the exposure phase, these times were compared between treatment groups (Fig. 4e). Interestingly, MAM rats actually spent significantly more time exploring the to-be familiar arm during the exposure phase [saline vs. MAM: $p<0.05]$. Thus, the deficit was not due to less exposure to the to-be familiar arm in the MAM group during the exposure phase.

Reduced anxiety levels in the elevated plus maze

Compared to saline controls, MAM rats showed reduced levels of anxiety as measured using the elevated plus maze (Fig. 5). The two sets of results were analysed together using ANOVAs with cohort (experimentally naïve versus watermaze experienced) and treatment condition (saline versus MAM) as between-subject factors. MAM rats made more entries and spent a higher proportion of time in the open arms of the maze compared to saline controls. Both entries and time open/closed arm ratios confirmed both a trend and a significant effect of treatment group, respectively [entries ratio: $F(1$, $52)=2.9, p<0.1$; time ratio: $F(1,52)=6.1, p<0.05]$. There was also a main effect of cohort (experimentally naïve versus watermaze experienced; [time ratio: $F(1,52)=27.6, p<0.001$; entries ratio: $F(1,52)=10.9, p<0.01])$. Experimentally naive rats spent a higher proportion of time in the closed arms and made fewer entries into the open arms of the maze compared to rats previously trained in the watermaze. However, importantly there was no group by cohort interaction $[p>0.1]$, therefore suggesting that previous watermaze experience may alter anxiety levels per se, but did not influence the effect of MAM treatment on anxiety levels on the elevated plus maze.

\section{Discussion}

The present data demonstrate that MAM E17 administration is sufficient to induce deficits in performance on associative, spatial reference memory tasks, both in the aversive hidden platform watermaze task and in the appetitive Y-maze task. In contrast, no or minor alterations were observed in the nonspatial T-maze visual discrimination learning task and the visible platform watermaze task, respectively. MAM rats were also impaired in the spatial novelty preference task which assesses short-term memory and exhibited reduced anxiety levels in the elevated plus maze. Overall, these findings are noteworthy for their demonstration of MAM E17-induced alterations in a wide range of dorsal and ventral hippocampusdependent behavioural tests. 
Fig. 4 MAM E17 exposure impaired spatial novelty preference in the Y-maze. Results are presented as the mean \pm SEM of the number of arm entries made (a) and time spent (b) in both the novel (blank) and familiar (striped) arms during the test phase, for both MAM E17 (grey, $n=14$ ) and saline controls (white, $n=12$ ). Difference (c) and ratio

(d) between the time spent in the novel versus familiar arm are also presented, as well as the time spent in the to-be familiar arm during the exposure phase (e). ${ }^{*} P<0.05$ vs. the saline-treated group
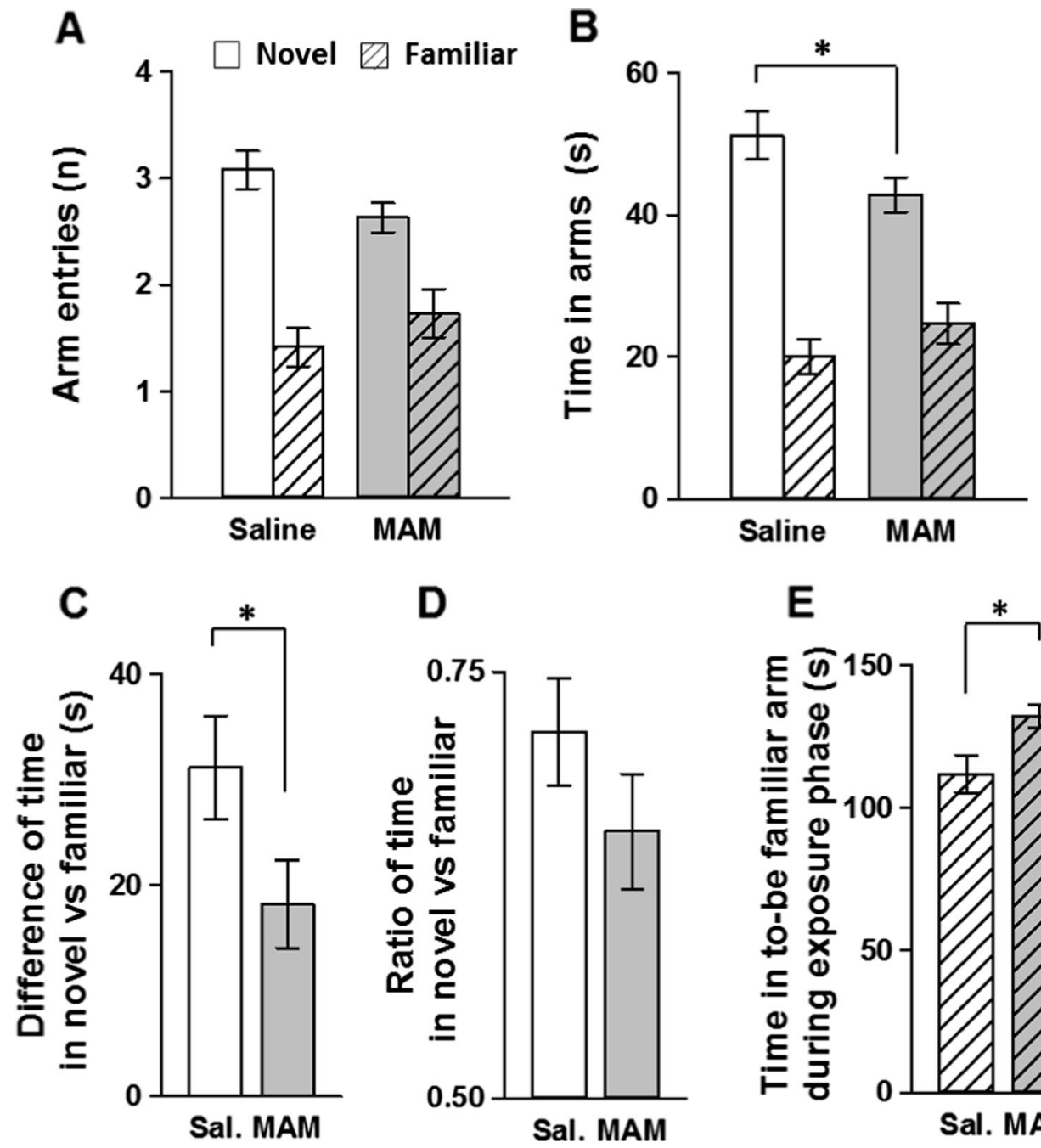

MAM E17 induced abnormal spatial processing

Despite strong evidence highlighting the anatomical and physiological alterations observed in the hippocampus of MAM E17 rats, discrepancies exist in the behavioural literature using this model. The present findings corroborate previous studies which have reported deficits in spatial working/short-term memory in these animals (Gourevitch et al. 2004; Featherstone et al. 2009; Hazane et al. 2009). We also demonstrate a robust deficit in spatial reference memory performance in MAM E17 rats in the Morris watermaze task, a result which though in agreement with one previous study (Hazane et al. 2009), is at odds with another (Flagstad et al. 2005).

The reason for such discrepancies remains unclear. It is possible that strain differences could be important in determining the behavioural effects of MAM treatment on watermaze performance. The study of Flagstad et al. (2005), which reported no effect on watermaze acquisition in MAM E17 rats was performed with Wistar rats, whereas all of the other studies discussed here, including our present study, have been conducted in Sprague-Dawley rats. Another important factor could be differences in the watermaze paradigms used in different laboratories. The different studies described here, including our own study, have varied in a number of different ways, including the size of the pool (ranging from anywhere between 1 and $2 \mathrm{~m}$ in diameter), the training protocols used, and in terms of the extra-maze spatial cues available to the animals to solve the task. Indeed, it is very difficult, if not impossible, to standardize the allocentric spatial cues that are available to the animals in the watermaze task across different laboratories. Nevertheless, it is possible to assess and demonstrate the hippocampal dependency of a particular watermaze task. In this respect, it is important to point out that we have previously reported robust and reliable deficits with cytotoxic hippocampal lesions in rats in the very same watermaze apparatus, in the very same testing room, with the very same spatial cues as used here in the current MAM E17 study (e.g. Bannerman et al. 1999). Thus, we are confident that our watermaze paradigm is a truly hippocampus-dependent test of spatial memory. 
Fig. 5 MAM E17 exposure reduced anxiety levels in the elevated plus maze. Two separate cohorts of animals were assessed: experimentally naïve (MAM: grey, $n=16$; saline: white, $n=13$ ) and trained animals (MAM grey, $n=14$; saline white, $n=12$ ). Results are presented as the mean \pm SEM of the time spent (a) and number of arm entries made (b) in the open arms of the maze. The mean \pm SEM of the ratios of time spent (c) and arm entries (d) in open arms are also expressed. $* P<0.05$ vs. the saline-treated group. ${ }^{\# \#} P<0.01 ;{ }^{\# \#} P<0.001$ vs. the experimentally naïve cohort
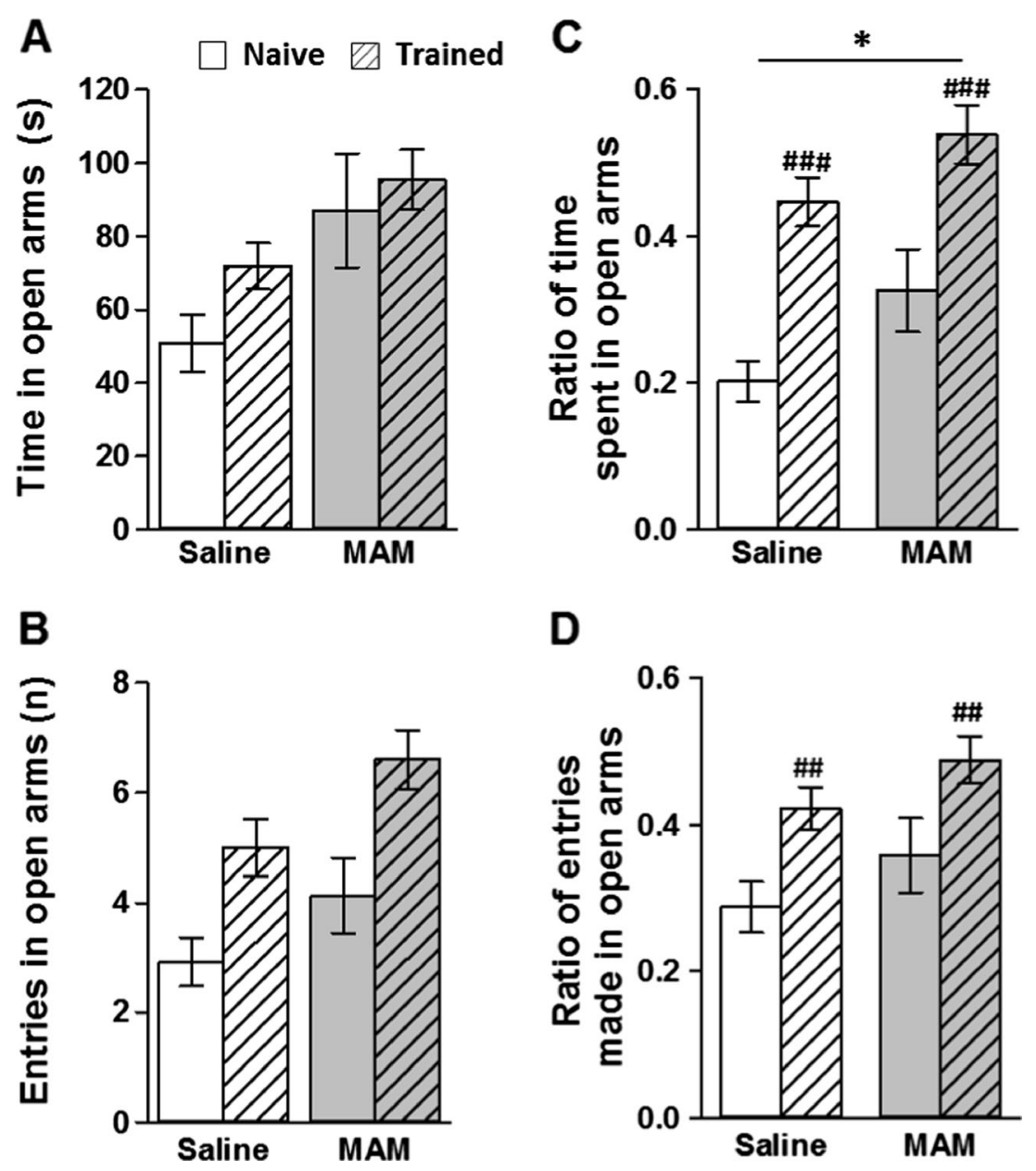

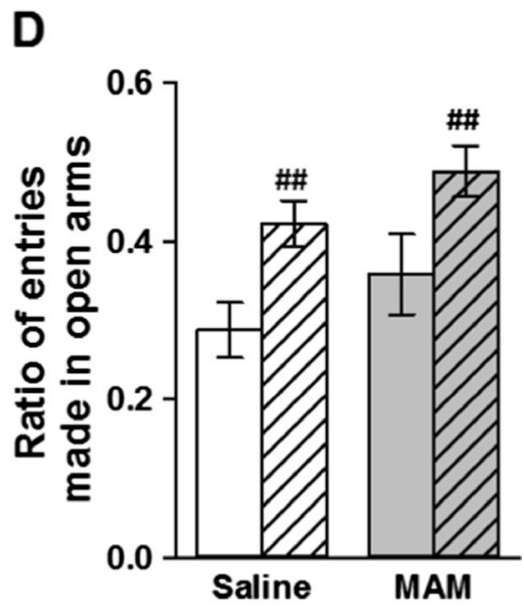

In addition, we have also shown that the spatial reference memory deficit extends to the non-aversive, appetitively motivated Y-maze task. MAM E17 rats also exhibited a shortterm spatial memory deficit on the Y-maze novelty preference task. These findings therefore demonstrate MAM E17induced deficits in several hippocampus-dependent memory tasks. However, it is also worth noting that, although significantly impaired in both the watermaze and appetitive Y-maze tasks, the MAM E17 rats are capable of demonstrating spatial learning in both paradigms. For example, after 32 watermaze training trials, the MAM E17 animals were showing a significant preference for the target quadrant during probe test 2 (spending almost $40 \%$ of time in the correct quadrant), albeit they were still impaired relative to controls. Moreover, the MAM E17 deficit in the appetitive Y-maze task is transient, with the two groups being indistinguishable after day 3 of training. Therefore, MAM treatment does not completely prevent hippocampus-dependent spatial learning. This is maybe not surprising given the more subtle effect of the MAM manipulation on hippocampal pathology and/or physiology, compared to a hippocampal lesion for example. Importantly, we found only minimal effects on non-spatial control tasks in the MAM E17 rats. Thus, the spatial memory deficits reported here are unlikely to reflect gross disturbances in sensorimotor or motivational processes which are necessary for task performance.

These MAM E17-induced deficits in spatial information processing are potentially in line with previous demonstrations of altered hippocampal pathology in these animals (Moore et al. 2006; Lodge and Grace. 2009; Hradetzky et al. 2012; Sanderson et al. 2012). However, it is also important to point out that we would not rule out the possibility of an extrahippocampal contribution to these spatial deficits, given the likely role that other brain areas, including cortex, play in spatial memory performance (see Bannerman et al. 2014 for discussion). Furthermore, it is also not clear which particular aspect (or aspects) of hippocampal physiology have been disrupted in order to produce these impairments. For example, recent proteomic and metabolic analyses of hippocampal slices from MAM E17 rats, identified changes in AMPA-R subunit expression and phosphorylation, suggesting synaptic transmission and plasticity may be altered in MAM rats, which could explain impairments in long-term spatial memory in these animals (Hradetzky et al. 2012). MAM E17-exposed animals also have significantly altered NMDA-R protein levels and function in hippocampus, both during juvenile 
and adolescent stages of development. These changes have been linked with learning and memory deficits in the Morris Watermaze (Snyder et al. 2013), although the contribution that hippocampal NMDARs make, if any, to spatial memory performance in the watermaze in adulthood is still a matter of debate (see Bannerman et al. 2014 for discussion). Moreover, both the dorsal and ventral hippocampal subregions in adult MAM E17 rats display increased excitability/ activity, reduced synaptic transmission and altered depotentiation (Lodge and Grace. 2007; Sanderson et al. 2012). Some or all of these physiological phenotypes could contribute to the behavioural phenotype that we have observed.

It is also of note that MAM E17 rats are more susceptible to stress-induced alterations in synaptic plasticity and behaviour (Goto and Grace. 2006; Sanderson et al. 2012; Du and Grace. 2013; Zimmerman et al. 2013). Along these lines, it is interesting to note that the MAM E17-induced spatial deficits were far greater in the Morris watermaze task compared to the appetitive Y-maze task. We acknowledge (i) that it is very difficult to compare across different measures of performance in different tasks, (ii) that there are various other important procedural differences between these two tasks which could explain differences in the magnitude of these effects and (iii) that they likely depend on different psychological processes to some extent (Bannerman et al. 2012). Nevertheless, these results are consistent with the possibility that the MAM E17 deficits might be, at least partly, dependent upon the levels of stress and/or arousal that the animals are subjected to during task performance.

\section{MAM E17 reduced anxiety levels}

Alongside its role in spatial information processing, the hippocampus has long been associated with aspects of emotionality and, in particular, with anxiety (Gray 1982; Gray and McNaughton. 2000). Anxiety is associated with conflict or uncertainty, and has evolved to prevent the subject from going into potentially dangerous situations. Rodent tests of anxiety, such as the elevated plus maze, are based on an approach versus avoidance conflict with the animal being required to choose whether to explore the open, exposed arms of the maze (approach), or to stay in the safe, enclosed sections (avoidance). In the present study, MAM E17 rats spent a greater amount of time and made more entries into the open arms of the elevated plus maze compared to saline controls, consistent with reduced anxiety in these animals, and highlighting their increased tendency to approach and explore an uncertain (and hence potentially dangerous) environment. This subtle but significant reduction in anxiety is in direct contrast with recent studies suggesting elevated anxiety levels and abnormal stress responsivity in adolescent MAM E17 rats. Indeed, Du and Grace (2013) found increased (rather than decreased) anxiety on the elevated plus maze in MAM E17 rats when tested in adolescence. Thus, the anxiety phenotype in MAM rats may be dependent on the age of the animals, which is potentially consistent with the hypothesis that these animals are more sensitive to the effects of stress in their youth. For example, Zimmerman et al. (2013) have shown an increased stress response in MAM E17 animals compared to controls in juvenile animals, but found that this effect was not present in older animals. Further studies are required to understand better how different aspects of emotionality are affected at different developmental stages in the MAM model, and how these phenotypes interact with the stress response.

We also cannot rule out the possibility that deficits in spatial memory abilities, and their resulting effects on spatial exploration, could influence the behaviour of the MAM animals on the elevated plus maze (both in this study and also elsewhere). The time spent in the open and closed arms of the elevated plus maze will, in part, reflect the relative ability of the different sections of the maze to induce exploration. This will, of course, depend on the salience of the different sections, the animals' starting location, and the rate of habituation in the different experimental groups. The latter may of course depend on the spatial memory abilities of the rats. Thus, there could be a complex set of interactions between different psychological processes during elevated plus maze performance, which might also vary from one laboratory to the next, depending the dimensions and character of the maze, the extramaze cues available, and the precise testing protocol adopted (see also Fitzgerald et al. 2010 for discussion). Against this, it is also worth pointing out that rats with dorsal hippocampal lesions display very pronounced spatial memory impairments but can behave normally on such approach/avoidance conflict tests (Kjelstrup et al. 2002; McHugh et al. 2004; but see also Bannerman et al. 2002).

Conversely, we cannot completely rule out the possibility that the spatial memory impairments may in some way reflect the reduced anxiety levels in the MAM E17 rats. However, the nature and direction of any interaction between anxiety levels and spatial memory performance in the watermaze is difficult to predict. On the one hand, one might argue that reduced anxiety levels might lead to less motivation to escape from the water but, equally, one might argue that reduced anxiety might result in a reduced stress response and it is well established that high levels of stress can disrupt hippocampus-dependent learning (Sandi and Pinelo-Nava. 2007). Notably, rats treated with an anxiolytic dose of the benzodiazepine chlordiazepoxide are impaired in the watermaze (McNaughton and Morris. 1987). In contrast, rats with ventral hippocampal lesions, which also display reduced anxiety (e.g. Bannerman et al. 2002, 2004; Kjelstrup et al. 2002; McHugh et al. 2004), are generally unaffected in the Morris watermaze task (see Moser et al. 1995; Bannerman et al. 1999, 2002; Kjelstrup et al. 2002). In fact, in one study, 
ventral hippocampal lesioned animals actually performed better than controls in a spatial reference memory version of the Morris watermaze task, similar to that used in the present study (Richmond et al. 1999).

Disentangling mnemonic effects from emotionality effects in the present watermaze study may be particularly difficult, given the subtle but significant deficit in the MAM E17 rats on the non-spatial, visible platform control task. In contrast, the spatial deficit in the appetitive Y-maze task was accompanied by normal performance on the appetitive, non-spatial visual discrimination maze task. These tasks are fairly well matched for sensorimotor and motivational demands, and there is no reason to think that they will differ in the levels of anxiety that they might generate. Although, it is important to point out that the order of testing was not counterbalanced which could be an important factor. Thus, on the basis of these data, one might conclude that there is a genuine spatial memory deficit in these animals which cannot be attributed to reduced levels of anxiety.

Of course the fact that MAM E17 rats display reduced anxiety on the elevated plus maze is not obviously consistent with what is seen in schizophrenic patients, with increased anxiety often acting as a trigger for psychotic episodes (Lysaker and Salyers. 2007). Moreover, anxiety disorders, featuring increased anxiety, show a high comorbidity with schizophrenia (Achim et al. 2011). Therefore, it remains important to investigate further how apparently similar morphological changes may trigger different behavioural/emotional phenotypes in MAM E17 rats compared to schizophrenic patients.

The present findings are however more generally consistent with a ventral hippocampal dysfunction in these animals. MAM E17 rats display anatomical and neurophysiological changes in the ventral part of the hippocampus (Sanderson et al. 2012). Increasing evidence suggests that the spatial memory and anxiety functions of the hippocampus are preferentially associated with its dorsal and ventral subregions, respectively (Moser et al. 1995; Bannerman et al. 2004, 2014; Fanselow and Dong. 2010). Ventral hippocampal lesions have been found to reduce anxiety on a number of ethologically based tests including the elevated plus maze test (Bannerman et al. 2002, 2003; Kjelstrup et al. 2002). However, determining which aspect or aspects of ventral hippocampal dysfunction are important, and how they determine the directionality of any effect on anxiety (anxiogenic versus anxiolytic) requires further study.

\section{Conclusions-MAM E17 as a model of hippocampal dysfunction in schizophrenia}

Offspring of rats dosed with MAM at E17 showed robust deficits in a wide range of hippocampus-dependent behavioural assays, including several spatial memory tasks and the elevated plus maze test of anxiety. While these behavioural phenotypes likely reflect, at least in part, the well-documented changes in hippocampal physiology reported previously in these animals, their relationship to the symptoms seen in schizophrenic patients remains unclear, particularly in terms of any emotionality phenotypes. Further studies are required to understand better these changes in emotionality induced by pre-natal MAM treatment, and to determine whether differential responses to stress and/or arousal could also impact on the cognitive phenotypes observed.

Financial disclosure Francois Gastambide and Mark Tricklebank declare being employees of Eli Lilly \& Co Ltd. Heta Svard and Sanna K Janhunen declare being employees of Orion Pharma. The research leading to these results has received support from the Innovative Medicines Initiative Joint Undertaking under grant agreement no. 115008 of which resources are composed of EFPIA in-kind contribution and financial contribution from the European Union's Seventh Framework Programme (FP7/2007-2013).

\section{References}

Achim AM, Bertrand MC, Sutton H, Montoya A, Czechowska Y, Malla AK, Joober R, Pruessner JC, Lepage M (2007) Selective abnormal modulation of hippocampal activity during memory formation in first-episode psychosis. Arch Gen Psychiatry 64:999-1014

Achim AM, Maziade M, Raymond E, Olivier D, Merette C, Roy MA (2011) How prevalent are anxiety disorders in schizophrenia? A meta-analysis and critical review on a significant association. Schizophr Bull 37:811-821. doi:10.1093/schbul/sbp148

Aleman A, Hijman R, de Haan EH, Kahn RS (1999) Memory impairment in schizophrenia: a meta-analysis. Am J Psychiatry 156:1358-1366

Antonova E, Sharma T, Morris R, Kumari V (2004) The relationship between brain structure and neurocognition in schizophrenia: a selective review. Schizophr Res 70:117-145. doi:10.1016/j.schres. 2003.12.002

Bannerman DM, Yee BK, Good MA, Heupel MJ, Iversen SD, Rawlins JN (1999) Double dissociation of function within the hippocampus: a comparison of dorsal, ventral, and complete hippocampal cytotoxic lesions. Behav Neurosci 113:1170-1188

Bannerman DM, Deacon RM, Offen S, Friswell J, Grubb M, Rawlins JN (2002) Double dissociation of function within the hippocampus: spatial memory and hyponeophagia. Behav Neurosci 116:884-901

Bannerman DM, Grubb M, Deacon RM, Yee BK, Feldon J, Rawlins JN (2003) Ventral hippocampal lesions affect anxiety but not spatial learning. Behav Brain Res 139:197-213

Bannerman DM, Rawlins JN, McHugh SB, Deacon RM, Yee BK, Bast T, Zhang WN, Pothuizen HH, Feldon J (2004) Regional dissociations within the hippocampus - memory and anxiety. Neurosci Biobehav Rev 28:273-283. doi:10.1016/j.neubiorev.2004.03.004

Bannerman DM, Bus T, Taylor A, Sanderson DJ, Schwarz I, Jensen V, Hvalby O, Rawlins JN, Seeburg PH, Sprengel R (2012) Dissecting spatial knowledge from spatial choice by hippocampal NMDA receptor deletion. Nat Neurosci 15:1153-1159. doi:10.1038/nn.3166

Bannerman DM, Sprengel R, Sanderson DJ, McHugh SB, Rawlins JN, Monyer H, Seeburg PH (2014) Hippocampal synaptic plasticity, spatial memory and anxiety. Nat Rev Neurosci 15:181-192. doi: $10.1038 / \mathrm{nrn} 3677$ 
Boyer P, Phillips JL, Rousseau FL, Ilivitsky S (2007) Hippocampal abnormalities and memory deficits: new evidence of a strong pathophysiological link in schizophrenia. Brain Res Rev 54:92-112

Chin CL, Curzon P, Schwartz AJ, O'Connor EM, Rueter LE, Fox GB, Day M, Basso AM (2011) Structural abnormalities revealed by magnetic resonance imaging in rats prenatally exposed to methylazoxymethanol acetate parallel cerebral pathology in schizophrenia. Synapse 65:393-403. doi:10.1002/syn.20857

Cirillo MA, Seidman LJ (2003) Verbal declarative memory dysfunction in schizophrenia: from clinical assessment to genetics and brain mechanisms. Neuropsychol Rev 13:43-77

Du Y, Grace AA (2013) Peripubertal diazepam administration prevents the emergence of dopamine system hyperresponsivity in the MAM developmental disruption model of schizophrenia. Neuropsychopharmacology 38:1881-1888. doi:10.1038/npp.2013. 101

Fanselow MS, Dong HW (2010) Are the dorsal and ventral hippocampus functionally distinct structures? Neuron 65:7-19. doi:10.1016/j. neuron.2009.11.031

Featherstone RE, Burton CL, Coppa-Hopman R, Rizos Z, Sinyard J, Kapur S, Fletcher PJ (2009) Gestational treatment with methylazoxymethanol (MAM) that disrupts hippocampaldependent memory does not alter behavioural response to cocaine. Pharmacol Biochem Behav 93:382-390. doi:10.1016/j.pbb.2009. 05.010

Fitzgerald PJ, Barkus C, Feyder M, Wiedholz LM, Chen YC, Karlsson RM, Machado-Vieira R, Graybeal C, Sharp T, Zarate C, HarveyWhite J, Du J, Sprengel R, Gass P, Bannerman D, Holmes A (2010) Does gene deletion of AMPA GluA1 phenocopy features of schizoaffective disorder? Neurobiol Dis 40:608-621. doi:10.1016/ j.nbd.2010.08.005

Flagstad P, Glenthoj BY, Didriksen M (2005) Cognitive deficits caused by late gestational disruption of neurogenesis in rats: a preclinical model of schizophrenia. Neuropsychopharmacology 30:250-260

Folley BS, Astur R, Jagannathan K, Calhoun VD, Pearlson GD (2010) Anomalous neural circuit function in schizophrenia during a virtual Morris water task. Neuroimage 49:3373-3384. doi:10.1016/j. neuroimage.2009.11.034

Gao XM, Sakai K, Roberts RC, Conley RR, Dean B, Tamminga CA (2000) Ionotropic glutamate receptors and expression of $N$-methylD-aspartate receptor subunits in subregions of human hippocampus: effects of schizophrenia. Am J Psychiatry 157:1141-1149

Goto Y, Grace AA (2006) Alterations in medial prefrontal cortical activity and plasticity in rats with disruption of cortical development. Biol Psychiatry 60:1259-1267

Gourevitch R, Rocher C, Le Pen G, Krebs MO, Jay TM (2004) Working memory deficits in adult rats after prenatal disruption of neurogenesis. Behav Pharmacol 15:287-292

Gray J (1982) The neuropsychology of anxiety: an enquiry into the functions of the septo-hippocampal system. Oxford University Press

Gray J, McNaughton N (2000) The neuropsychology of anxiety: an enquiry into the functions of the septo-hippocampal system - second edition. Oxford University Press

Hanlon FM, Weisend MP, Hamilton DA, Jones AP, Thoma RJ, Huang M, Martin K, Yeo RA, Miller GA, Canive JM (2006) Impairment on the hippocampal-dependent virtual Morris water task in schizophrenia. Schizophr Res 87:67-80

Harrison PJ (2004) The hippocampus in schizophrenia: a review of the neuropathological evidence and its pathophysiological implications. Psychopharmacology (Berl) 174:151-162. doi:10.1007/s00213003-1761-y

Harrison PJ, Law AJ, Eastwood SL (2003) Glutamate receptors and transporters in the hippocampus in schizophrenia. Ann N Y Acad Sci 1003:94-101
Hazane F, Krebs MO, Jay TM, Le Pen G (2009) Behavioral perturbations after prenatal neurogenesis disturbance in female rat. Neurotox Res 15:311-320. doi:10.1007/s12640-009-9035-Z

Heckers S, Konradi C (2002) Hippocampal neurons in schizophrenia. J Neural Transm 109:891-905. doi:10.1007/s007020200073

Heckers S, Rauch SL, Goff D, Savage CR, Schacter DL, Fischman AJ, Alpert NM (1998) Impaired recruitment of the hippocampus during conscious recollection in schizophrenia. Nat Neurosci 1:318-323. doi:10.1038/1137

Heinrichs RW, Zakzanis KK (1998) Neurocognitive deficit in schizophrenia: a quantitative review of the evidence. Neuropsychology 12: 426-445

Honea R, Crow TJ, Passingham D, Mackay CE (2005) Regional deficits in brain volume in schizophrenia: a meta-analysis of voxel-based morphometry studies. Am J Psychiatry 162: 2233-2245

Hradetzky E, Sanderson TM, Tsang TM, Sherwood JL, Fitzjohn SM, Lakics V, Malik N, Schoeffmann S, O’Neill MJ, Cheng TM, Harris LW, Rahmoune H, Guest PC, Sher E, Collingridge GL, Holmes E, Tricklebank MD, Bahn S (2012) The methylazoxymethanol acetate (MAM-E17) rat model: molecular and functional effects in the hippocampus. Neuropsychopharmacology 37:364-377. doi:10.1038/npp. 2011.219

Jessen F, Scheef L, Germeshausen L, Tawo Y, Kockler M, Kuhn KU, Maier W, Schild HH, Heun R (2003) Reduced hippocampal activation during encoding and recognition of words in schizophrenia patients. Am J Psychiatry 160:1305-1312

Kjelstrup KG, Tuvnes FA, Steffenach HA, Murison R, Moser EI, Moser MB (2002) Reduced fear expression after lesions of the ventral hippocampus. Proc Natl Acad Sci U S A 99:10825-10830. doi:10. 1073/pnas.152112399

Kovelman JA, Scheibel AB (1984) A neurohistological correlate of schizophrenia. Biol Psychiatry 19:1601-1621

Lawrie SM, Abukmeil SS (1998) Brain abnormality in schizophrenia. A systematic and quantitative review of volumetric magnetic resonance imaging studies. $\mathrm{Br} \mathrm{J}$ Psychiatry 172: $110-120$

Le Pen G, Gourevitch R, Hazane F, Hoareau C, Jay TM, Krebs MO (2006) Peri-pubertal maturation after developmental disturbance: a model for psychosis onset in the rat. Neuroscience 143:395405

Leube DT, Rapp A, Buchkremer G, Bartels M, Kircher TT, Erb M, Grodd W (2003) Hippocampal dysfunction during episodic memory encoding in patients with schizophrenia-an fMRI study. Schizophr Res 64:83-85

Lodge DJ, Grace AA (2007) Aberrant hippocampal activity underlies the dopamine dysregulation in an animal model of schizophrenia. J Neurosci 27:11424-11430

Lodge DJ, Grace AA (2009) Gestational methylazoxymethanol acetate administration: a developmental disruption model of schizophrenia. Behav Brain Res 204:306-312. doi:10.1016/j.bbr.2009.01.031

Lysaker PH, Salyers MP (2007) Anxiety symptoms in schizophrenia spectrum disorders: associations with social function, positive and negative symptoms, hope and trauma history. Acta Psychiatr Scand 116:290-298

Matricon J, Bellon A, Frieling H, Kebir O, Le Pen G, Beuvon F, DaumasDuport C, Jay TM, Krebs MO (2010) Neuropathological and Reelin deficiencies in the hippocampal formation of rats exposed to MAM; differences and similarities with schizophrenia. PLoS One 5: e10291. doi:10.1371/journal.pone.0010291

McHugh SB, Deacon RM, Rawlins JN, Bannerman DM (2004) Amygdala and ventral hippocampus contribute differentially to mechanisms of fear and anxiety. Behav Neurosci 118:63-78. doi: 10.1037/0735-7044.118.1.63 
McNaughton N, Morris RG (1987) Chlordiazepoxide, an anxiolytic benzodiazepine, impairs place navigation in rats. Behav Brain Res 24: 39-46. doi:10.1016/0166-4328(87)90034-9

Medoff DR, Holcomb HH, Lahti AC, Tamminga CA (2001) Probing the human hippocampus using rCBF: contrasts in schizophrenia. Hippocampus 11:543-550. doi:10.1002/hipo.1070

Moore H, Jentsch JD, Ghajarnia M, Geyer MA, Grace AA (2006) A neurobehavioral systems analysis of adult rats exposed to methylazoxymethanol acetate on E17: implications for the neuropathology of schizophrenia. Biol Psychiatry 60:253-264

Moser MB, Moser EI, Forrest E, Andersen P, Morris RG (1995) Spatial learning with a minislab in the dorsal hippocampus. Proc Natl Acad Sci U S A 92:9697-9701

Nelson MD, Saykin AJ, Flashman LA, Riordan HJ (1998) Hippocampal volume reduction in schizophrenia as assessed by magnetic resonance imaging: a meta-analytic study. Arch Gen Psychiatry 55: 433-440

Nuechterlein KH, Barch DM, Gold JM, Goldberg TE, Green MF, Heaton RK (2004) Identification of separable cognitive factors in schizophrenia. Schizophr Res 72:29-39

Ongur D, Cullen TJ, Wolf DH, Rohan M, Barreira P, Zalesak M, Heckers $S$ (2006) The neural basis of relational memory deficits in schizophrenia. Arch Gen Psychiatry 63:356-365

Penschuck S, Flagstad P, Didriksen M, Leist M, Michael-Titus AT (2006) Decrease in parvalbumin-expressing neurons in the hippocampus and increased phencyclidine-induced locomotor activity in the rat methylazoxymethanol (MAM) model of schizophrenia. Eur J Neurosci 23:279-284

Phillips KG, Bartsch U, McCarthy AP, Edgar DM, Tricklebank MD, Wafford KA, Jones MW (2012a) Decoupling of sleep-dependent cortical and hippocampal interactions in a neurodevelopmental model of schizophrenia. Neuron 76:526-533. doi:10.1016/j. neuron.2012.09.016

Phillips KG, Cotel MC, McCarthy AP, Edgar DM, Tricklebank M, O'Neill MJ, Jones MW, Wafford KA (2012b) Differential effects of NMDA antagonists on high frequency and gamma EEG oscillations in a neurodevelopmental model of schizophrenia. Neuropharmacology 62:1359-1370. doi:10.1016/j.neuropharm. 2011.04.006

Reichenberg A, Harvey PD (2007) Neuropsychological impairments in schizophrenia: integration of performance-based and brain imaging findings. Psychol Bull 133:833-858

Richmond MA, Yee BK, Pouzet B, Veenman L, Rawlins JN, Feldon J, Bannerman DM (1999) Dissociating context and space within the hippocampus: effects of complete, dorsal, and ventral excitotoxic hippocampal lesions on conditioned freezing and spatial learning. Behav Neurosci 113:1189-1203. doi:10.1037/0735-7044.113.6. 1189

Sanderson TM, Cotel MC, O’Neill MJ, Tricklebank MD, Collingridge GL, Sher E (2012) Alterations in hippocampal excitability, synaptic transmission and synaptic plasticity in a neurodevelopmental model of schizophrenia. Neuropharmacology 62:1349-1358. doi:10.1016/ j.neuropharm.2011.08.005

Sandi C, Pinelo-Nava MT (2007) Stress and memory: behavioral effects and neurobiological mechanisms. Neural Plast 2007:78970. doi:10. 1155/2007/78970

Schobel SA, Lewandowski NM, Corcoran CM, Moore H, Brown T, Malaspina D, Small SA (2009) Differential targeting of the CA1 subfield of the hippocampal formation by schizophrenia and related psychotic disorders. Arch Gen Psychiatry 66:938-946. doi:10.1001/ archgenpsychiatry.2009.115

Snyder MA, Adelman AE, Gao WJ (2013) Gestational methylazoxymethanol exposure leads to NMDAR dysfunction in hippocampus during early development and lasting deficits in learning. Neuropsychopharmacology 38:328-340. doi:10.1038/npp. 2012.180

Stone WS, Hsi X (2011) Declarative memory deficits and schizophrenia: problems and prospects. Neurobiol Learn Mem 96:544-552. doi:10. 1016/j.nlm.2011.04.006

Tamminga CA, Stan AD, Wagner AD (2010) The hippocampal formation in schizophrenia. Am J Psychiatry 167:1178-1193. doi:10.1176/ appi.ajp.2010.09081187

Tsai G, Passani LA, Slusher BS, Carter R, Baer L, Kleinman JE, Coyle JT (1995) Abnormal excitatory neurotransmitter metabolism in schizophrenic brains. Arch Gen Psychiatry 52:829-836

Weiss AP, Heckers S (2001) Neuroimaging of declarative memory in schizophrenia. Scand J Psychol 42:239-250

Wright IC, Rabe-Hesketh S, Woodruff PW, David AS, Murray RM, Bullmore ET (2000) Meta-analysis of regional brain volumes in schizophrenia. Am J Psychiatry 157:16-25

Zhang ZJ, Reynolds GP (2002) A selective decrease in the relative density of parvalbumin-immunoreactive neurons in the hippocampus in schizophrenia. Schizophr Res 55:1-10

Zimmerman EC, Bellaire M, Ewing SG, Grace AA (2013) Abnormal stress responsivity in a rodent developmental disruption model of schizophrenia. Neuropsychopharmacology 38:2131-2139. doi:10. 1038/npp.2013.110 